EPJ manuscript No.

(will be inserted by the editor)

\title{
Quantum state transfer and time-dependent disorder in Quantum Chains
}

\author{
Daniel Burgarth \\ Institute of Theoretical Computer Science, ETH Zurich,8092 Zurich, Switzerland
}

\begin{abstract}
One of the most basic tasks required for Quantum Information Technology is the ability to connect different components of a Quantum Computer by quantum wires that obey the superposition principle. Since superpositions can be very sensitive to noise this turns out to be already quite difficult. Recently, it was suggested to use chains of permanently coupled spin- $1 / 2$ particles (quantum chains) for this purpose. They have the advantage that no external control along the wire is required during the transport of information, which makes it possible to isolate the wire from sources of noise. We first give an introduction to basic quantum state transfer and review existing advanced schemes by other authors. We then show a new result that demonstrates the stability of the scheme [1] against disorder that is approximately constant during one application of the channel, but time-dependent with respect to multiple applications.
\end{abstract}

\section{Introduction}

\subsection{Spin chains for quantum state transfer}

It is perhaps surprising that more than twenty years after the theoretical birth of Quantum Computers only very small prototypes have been built. One of the main problems is the "programming", i.e. the design of a specific (time-dependent) Hamiltonian, usually described as a set of discrete unitary gates. This turns out to be extremely difficult because we need to connect microscopic objects (those behaving quantum mechanically) with macroscopic devices that control the microscopic behavior. Even if one manages to find a link between the micro- and the macroscopic world, such as laser pulses and electric or magnetic fields, the connection often introduces not only control but also noise (dissipation and decoherence [2]) to the microscopic system, and its quantum behavior is diminished.

As part of the vision to develop theoretical methods narrowing the gap between what is imagined theoretically and what can be done experimentally, S. Bose suggested to use chains of permanently coupled quantum systems for the specific task of quantum communication 3 . Due to the "always on" coupling, these devices can in principle be built in such a way that they do not require external control to perform their tasks, just like a mechanical clockwork. This also overcomes the problem of decoherence as they can be separated from any source of noise. Although in theory, the universal set of gates on the quantum computer can be used to transfer quantum states by applying sequences of two-qubit swap gates (Fig. 1), in practice it is crucial to minimize the required number of quantum gates, as each gate typically introduces errors. In this light it appears costly to perform $N-1$ swap gates between nearest neighbors to just move a qubit state over a distance of $N$ sites. For example, Shor's algorithm on $N$ qubits can be implemented by only $\log N$ quantum gating operations [4] if long distant qubit gates are available. These long distant gates could consist of local gates followed by a quantum state transfer. If however the quantum state transfer is implemented as a sequence of local gates, then 


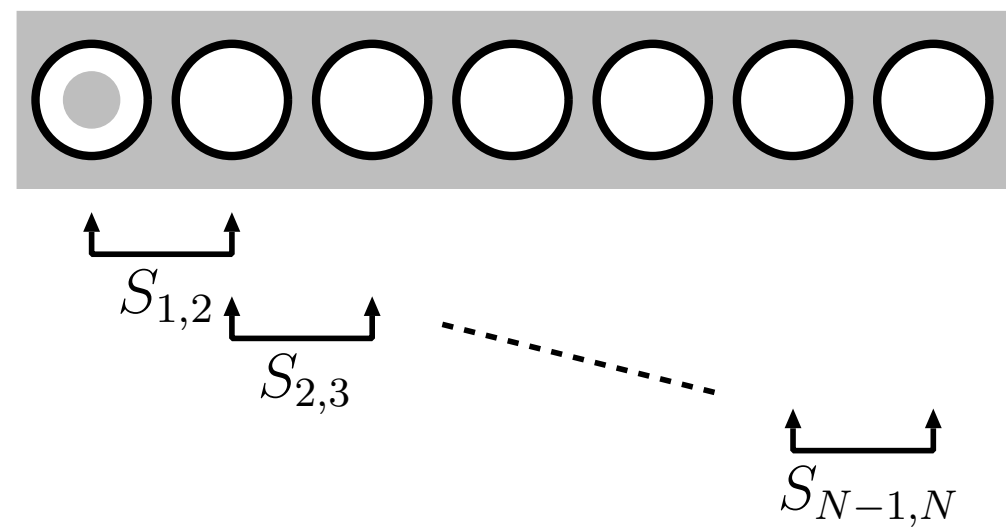

Fig. 1. In areas of universal control, quantum states can easily be transferred by sequences of unitary swap gates $S_{j, k}$ between nearest neighbours.

the number of operations blows up to the order of $N$ gates. The quantum state transfer can even be thought of as the source of the power of quantum computation, as any quantum circuit with $\log N$ gates and local gates only can be efficiently simulated on a classical computer 566.

A second reason to consider devices for quantum state transfer is related to scalability. While small quantum computers have already been built [7, it is very difficult to build large arrays of fully controllable qubits. A black box that transports unknown quantum states could be used to build larger quantum computers out of small components by connecting them. Likewise, quantum state transfer can be used to connect different components of a quantum computer, such as the processor and the memory. On larger distances, flying qubits such as photons, ballistic electrons and guided atoms/ions are considered for this purpose [89. However, converting back and forth between stationary qubits and mobile carriers of quantum information and interfacing between different physical implementations of qubits is very difficult and not worthy only for short communication distances. This is the typical situation one has to face in solid state systems, where quantum information is usually contained in the states of fixed objects such as quantum dots or Josephson junctions. In many cases, permanent couplings (and hence quantum chains) are easy to build in solid state devices. The qubits can be of the same type as the other qubits in the device, so no interfacing is required.

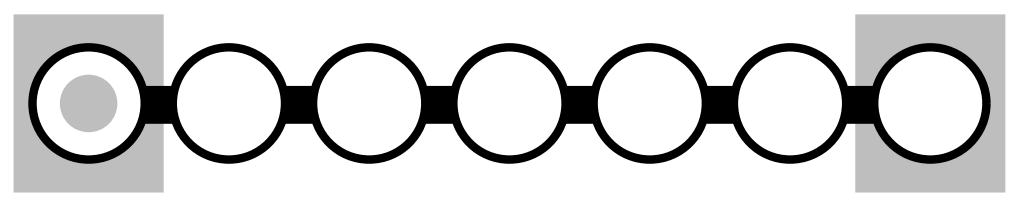

Fig. 2. Permanently coupled quantum chains can transfer quantum states without control along the line. Note that the ends still need to be controllable to initialize and read out quantum states.

Finally, an important reason to study quantum state transfer in quantum chains stems from a more fundamental point of view. Such systems in principle allow tests of Bell-inequalities and non-locality in solid-state experiments well before the realization of a full quantum computer. Although quantum transport is quite an established field, the quantum information point of view offers many new perspectives. Here, one looks at the transport of information rather than excitations, and at entanglement [10/11/12/13] rather than correlation functions. It has recently been shown that this sheds new light on well-known physical phenomena such as quantum phase transitions [14|15|16|17, quantum chaos [18|19|20|21] and localization [22|23|24]. Furthermore, quantum information takes on a more active attitude. The correlations of the system are not just calculated, but one also looks at how they may be changed. 


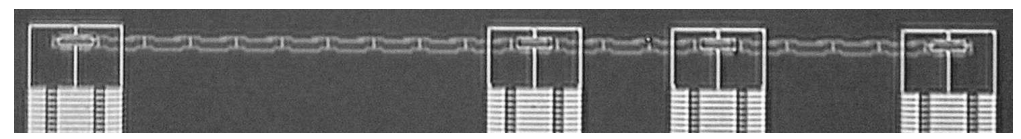

Fig. 3. A quantum chain consisting of $N=20$ flux qubits 29] (picture and experiment by Floor Paauw, TU Delft). The chain is connected to four larger SQUIDS for readout and gating.

\subsection{Physical implementations and experiments}

As we have seen above, the main advantage of state transfer with quantum chains is that the qubits can be of the same type as those used for the quantum computation. Therefore, most systems that are thought of as possible realizations of a quantum computer can also be used to build quantum chains. Of course there has to be some coupling between the qubits. This is typically easy to achieve in solid state systems, such as Josephson junctions with charge qubits 25 26 27], flux qubits 28,29] (see also Fig. 3) or quantum dots dots using the electrons 3031 or excitons 3233 as qubits. Other systems where quantum chain Hamiltonians can at least be simulated are NMR qubits 343536 37] and optical lattices 38. Such a simulation is particularly useful in the latter case, where local control is extremely difficult. Finally, qubits in cavities [3940] and coupled arrays of cavities were considered 4142 43]. For more fundamental questions, such as studies of entanglement transfer, non-locality and coherent transport, the quantum chains could also be realized by systems which are not typically thought of as qubits, but which are natural spin chains. These can be molecular systems [44] or quasi-1D solid state materials [45 46].

\subsection{Excitation conserving chains and correlation functions}

Most investigations on quantum state transfer with quantum chains up to date are concentrating on Hamiltonians that conserve the number of excitations (some more general cases are considered in [14 152947]). These Hamiltonians are much easier to handle both analytically and numerically, and it is also easier to get an intuition of the dynamics. Furthermore, they occur quite naturally as a coupling between qubits in the relevant systems. We stress though that there is no fundamental reason to restrict quantum chain communication to this case. We denote the eigenstates of the Pauli matrix $\sigma_{z}$ as $\sigma_{z}|0\rangle=|0\rangle$ and $\sigma_{z}|1\rangle=-|1\rangle$. The conservation of excitations means that $\sum_{k} \sigma_{z}^{(k)}$ commutes with the Hamiltonian $H$ of the system, where $\sigma_{z}^{(k)}$ is the Pauli matrix acting on the $k$ th qubit of the chain. The state $|\mathbf{0}\rangle \equiv|0\rangle^{(1)} \otimes|0\rangle^{(2)} \otimes \cdots \otimes|0\rangle^{(N)}$ with zero excitations is always an eigenstate of $H$. We assume that the system is initially in this state, which could be the case if it is the ground state (as in the ferromagnetic case or in the presence of a strong field in $z$ direction), or which could be achieved actively using an appropriate cooling protocol [48950]. The original state transfer protocol then proceeds as follows:

1. The state of the first qubit of the chain is swapped with an arbitrary and unknown state $\rho$. This is assumed to happen much faster than the time-scale of the interaction of the chain. It has recently been shown 28 that this is not a fundamental restriction, and that finite switching times can even slightly improve the fidelity if they are carefully included in the protocol. But this requires to solve the full time-dependent Schrödinger equation, and introduces further parameters to the model (i.e. the raise and fall time of the couplings). For the sake of simplicity, we will therefore stick to the original assumption.

2. After time evolution $U=\exp (-i H t)$ for the time $t$ the quantum state at the end $N$ of the chain is picked up. Again this is assumed to happen very fast.

3. If the chain is used multiple times, it has to be brought back to the initial state $|\mathbf{0}\rangle$ in order to avoid memory effects [51].

The quality of the transfer is quantified by the fidelity between the input state $\rho$ and the state that is picked up at the receiving end. Since it still depends on the state $\rho$ that is being sent, 
one can either average over all input states, or consider its minimum over all input states. We are interested in the latter, which is easily seen [50] to be

$$
F_{0}(t)=\left|\left\langle\sigma_{-}^{(N)}(t) \sigma_{+}^{(1)}\right\rangle\right|^{2}
$$

where $\sigma_{-}^{(N)}(t)=U^{\dagger}(t) \sigma_{-}^{(N)} U(t)$ and the expectation value is taken in the state $|\mathbf{0}\rangle$. Eq. (1) provides an interesting and intuitive connection between the time dependent two-point correlation functions and state transfer for excitation conserving systems. In statistical mechanics, one is typically interested in the thermodynamic limit of these functions and in their scaling for long time and distance. In the context of state transfer, their short time behavior and the height and width of their maxima are the most interesting quantities. Typically, due to the dispersion on the chain, the fidelity of these devices is not good enough to make them feasible 3 352 153.

\subsection{Advanced schemes}

Shortly after the initial proposal [3] it has been shown that there are ways to achieve even perfect state transfer with arbitrarily long chains. The Heisenberg model chosen by Bose features many typical aspects of coherent transport, i.e. the wave-like behavior, the dispersion, and the almost-periodicity of the fidelity. These features do not depend so much on the specific choices of the parameters of the chain, such as the couplings strengths. There are however specific couplings for quantum chains that show a quite different time evolution, and it was suggested in 554 and independently in 55] to use these to achieve a perfect state transfer. These values for engineered couplings also appear in a different context in 56 57. The time evolution features an additional mirror symmetry: the wave-packet disperses initially, but the dispersion is reversed after its center has passed the middle of the chain. This approach has been extended by various authors $12 / 47 / 53 / 58|59| 60|6162| 63|64| 65|66| 67|68| 69|70| 71$, and many other choices of parameters for perfect or near perfect state transfer in various settings were found 666870 ] A different approach of tuning the Hamiltonian was suggested in [72/73/74. There, only the first and the last couplings $j$ of the chain are engineered to be much weaker than the remaining couplings $J$ of the chain, which can be quite arbitrary. The fidelity can be made arbitrarily high by making the edge coupling strengths weaker. Some specific types of quantum chains which show high fidelity for similar reasons were also investigated [75/76/77/78/79|80. The disadvantage of reliying on specific couplings strengths is that they the fidelity is typically not stable against disorder 268182.

A second approach is to encode the information in multiple qubits. It was suggested first in 52. There, it was assumed that the chain consists of three sections: one part controlled by the sending party, one "free" part and one part controlled by the receiving party. The sender encodes the qubit not only in a single qubit of the chain, but in a Gaussian-modulated superposition of his qubits. These Gaussian packets are known to have minimal dispersion. Likewise, the receiver performs a decoding operation on all qubits he controls. Near-perfect fidelity can be reached. Other strategies that involve multi-qubit encodings or decodings were suggested in 831848586

Finally, a number of authors found ways of improving the fidelity by time-dependent control of some parameters of the Hamiltonian. In 87] it is shown that if the end couplings can be controlled as arbitrary (in general complex valued) smooth functions of time the encoding scheme [52] could be simulated without the requirement of additional operations and qubits. Another possibility to achieve perfect state transfer is to have an Ising interaction with additionally pulsed global rotations 35 [88|89]. Further related methods of manipulating the transfer by global fields were reported in [18|2190|91|92 93 94 . 


\section{Optimal spin chain communication in the presence of coupling fluctuations}

\subsection{Setup}

In [1 it was shown that that the fidelity can be improved easily by applying in certain timeintervals two-qubit unitary gates at the receiving end of the chain. These gates act as valves which take probability amplitude out of the system without ever putting it back. We remark that this seeming time-irreversible behavior does not contradict quantum mechanics, because the gates depend on the step of the protocol. The required sequence is determined a priori by the Hamiltonian of the system and by the time-intervals of the protocol. Arbitrarily high fidelity is guaranteed by a convergence theorem for all non-Ising coupling types that conserve the number of excitations. This includes disordered systems, though the time-scale of the convergence will be very large if there is localization [24. For short chains or weak disorder however Anderson localization does not play any role and our protocol performs very stable - opposed to most other schemes. An experimental demonstration of this iterative approach has recently been given in a NMR system [37.

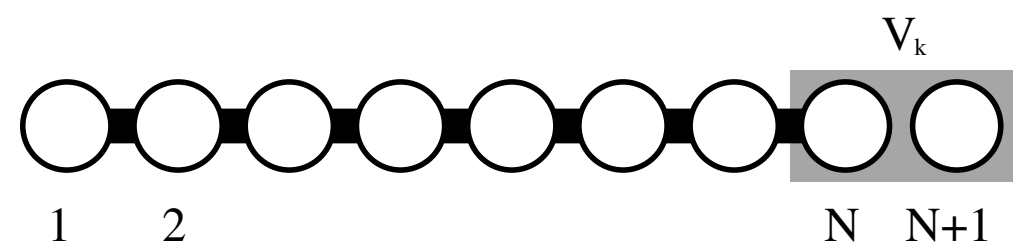

Fig. 4. A quantum chain (qubits $1,2, \cdots, N)$ and a target qubit $(N+1)$. By applying a sequence of two-qubit unitary gates $V_{k}$ on the last qubit of the chain and the target qubit, a state transfer of arbitrarily high fidelity can be achieved between the 1 st and $N+1$ th qubit.

We now very briefly describe the valve method from a practical point of view, i.e. how to determine the required operations from the Hamiltonian of the system. The proofs and details are described elsewhere [1]. Notice first that we consider $N+1$ qubits: the first $N$ are part of the chain, and the $N+1$ one is the target qubit to which we transfer. This qubit is not part of the chain and assumed to be uncoupled. The valve operations take place between the last qubit of the chain and the target qubit (see Fig. 4). We define the states $|\mathbf{n}\rangle \equiv \sigma_{+}^{(n)}|\mathbf{0}\rangle$ with exactly one excitation in the qubit $n$. The time interval between the $k$ th step and the $k-1$ th step of the protocol is $t_{k}$ with the corresponding time evolution operator of the chain $U_{k}=\exp \left(-i H t_{k}\right)$. Finally we define the unnormalized states $\left|\phi_{k}\right\rangle$ recursively by $\left|\phi_{k+1}\right\rangle=P U_{k}\left|\phi_{k}\right\rangle$ with $\left|\phi_{0}\right\rangle=|\mathbf{1}\rangle$ and $P=1-|\mathbf{N}\rangle\langle\mathbf{N}|$. The valve gates are two-qubit gates acting on qubits $N$ and $N+1$. Their ideal sequence is expressed in the canonical basis $\{|00\rangle,|01\rangle,|10\rangle,|11\rangle\}$ by

$$
V_{k}=\left(F_{0}^{k}\right)^{-1 / 2}\left(\begin{array}{cccc}
1 & 0 & 0 & 0 \\
0 & \left(F_{0}^{k-1}\right)^{-1 / 2} & \left\langle\mathbf{N}\left|U_{k}\right| \phi_{k-1}\right\rangle^{*} & 0 \\
0-\left\langle\mathbf{N}\left|U_{k}\right| \phi_{k-1}\right\rangle & \left(F_{0}^{k-1}\right)^{-1 / 2} & 0 \\
0 & 0 & 0 & 1
\end{array}\right),
$$

where the minimal fidelity for quantum state transfer at the $k$ th step of the protocol is given by

$$
F_{0}^{k}=1-\left\langle\phi_{k} \mid \phi_{k}\right\rangle .
$$

This is a generalization of the time-dependent two-point correlation function Eq. (1) in the presence of active gating. Ideally the gates act in a time-scale much shorter than the evolution of the chain, though this can be generalized [2811. For a given Hamiltonian one can easily compute the required $V_{k}$ as a function of the time interval $t_{k}$. The required optimization may take various constraints into account: low number of gates, high fidelity, short total time, regular intervals and stability with respect to timing fluctuations. 


\subsection{Fluctuations}

Now we consider the following: suppose one has computed a good sequence of gates $V_{k}$ for the Hamiltonian $H$ that one planned to implement. This could be an engineered Hamiltonian even in order to have a high initial fidelity. When the chain is built, its real Hamiltonian will be different from $H$ due to the finite precision of the implementation parameters (such as the distance of the qubits, electric or magnetic fields, and so on). This time-independent error can in principle be compensated by performing a simple type of state tomography 84. and by re-adjusting the gates $V_{k}$ to the new Hamiltonian. But what happens if there are time-dependent errors, caused by voltage fluctuations or small displacements of the qubits? Alternatively, what happens if -say in mass production- one is ignorant about even the static errors, and refuses to perform tomography and a re-adjusting of the gates for each individual chain? For these questions it is clearly interesting to study what happens if the sequence $V_{k}$ is applied to the wrong Hamiltonian. In principle, this could be a arbitrary time-dependent Hamiltonian. For simplicity we restrict ourselves to slowly varying Hamiltonians which are approximately constant during one application of the protocol, but which have changed when the chain is used the next time. This assumption allows us to solve the problem for timeindependent Hamiltonians and then average over different realizations. The gates $V_{k}$ now no longer function as valves: they also put probability amplitude from the target qubit back into the chain. Since they are defined recursively, it could be that the error is amplified during the protocol, and that the fidelity as a function of the number of steps $k$ will strongly decrease after some critical value.

For the numerical analysis, we have considered a $X X$ Hamiltonian

$$
H(\underline{\delta})=\sum_{n=1}^{N-1}\left(1+\delta_{n}\right)\left[\sigma_{x}^{(n)} \sigma_{x}^{(n+1)}+\sigma_{y}^{(n)} \sigma_{y}^{(n+1)}\right]
$$

with coupling fluctuations $\underline{\delta}=\left(\delta_{1}, \ldots, \delta_{N-1}\right)$. A good sequence of gates $V_{k}$ is determined numerically for the ideal case $\underline{\delta}=\underline{0}$. Then the protocol with these $V_{k}$ is applied to a perturbed Hamiltonian where the $\delta_{n}$ are uniformly distributed in the interval $[-\Delta, \Delta]$, and the resulting minimal fidelity is computed. This is repeated over 100 samples and averaged. Rather than a break-down of the fidelity after a critical number of steps, we observe a saturation: at some point, the gain of applying another gate is equal to the loss that is caused by the error (Fig. 5). A similar behavior was already observed in the case of a switchable interaction [1. It is then interesting to compute the maximum of $F_{0}^{k}$ with respect to $k$ and its dependence on the strength of the error. The results shown in Fig. 6 are very motivating: the decrease is almost linearly in $\Delta$ over a wide range. Moreover, our protocol is capable of improving the "natural" performance of the chain (given by Eq. (10) for fluctuations up to $\Delta=0.3$. We obtained similar results also for Gaussian distributed disorder and for on-site disorder.

\section{Conclusion}

We have shown numerically that the valve protocol is surprisingly robust with respect to known and unknown disorder (such as slowly variying time-dependent disorder). This is a strong advantage with respect to the dual-rail scheme 84 which is only capable of dealing with known disorder. It remains open to study in more detail time-dependent Hamiltonians and to take into account realistic gating times, though we do not expect a qualitative difference to the results discussed here.

I would like to thank the organizers of the 383. Wilhelm und Else Heraeus Seminar for inviting me to contribute to this stimulating workshop. I am very grateful to V. Giovannetti and S. Bose for the long-standing collaboration. The work is finacially supported by the Swiss National Science Foundation (SNSF). 


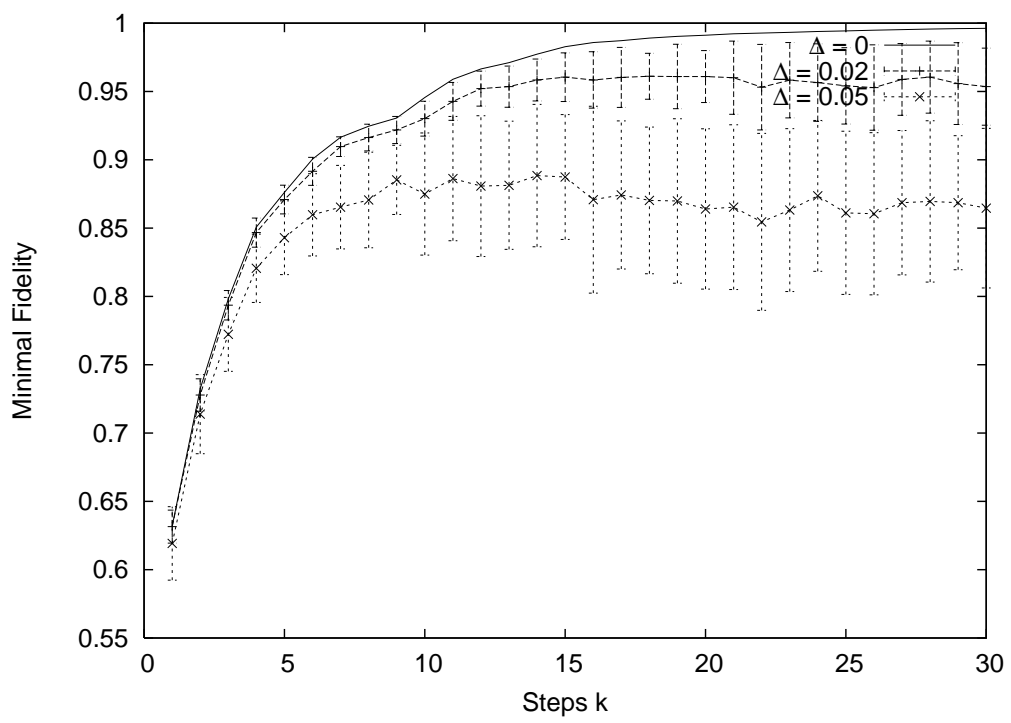

Fig. 5. Minimal fidelity $F_{0}^{k}$ for a $X X$ chain of length $N=20$ as a function of the steps $k$ of the protocol. Shown is the perfect case $(\Delta=0)$ and two perturbed systems with different fluctuation strength $(\Delta=0.02,0.05)$. In the latter cases, we give the average and standard deviation for 100 samples.

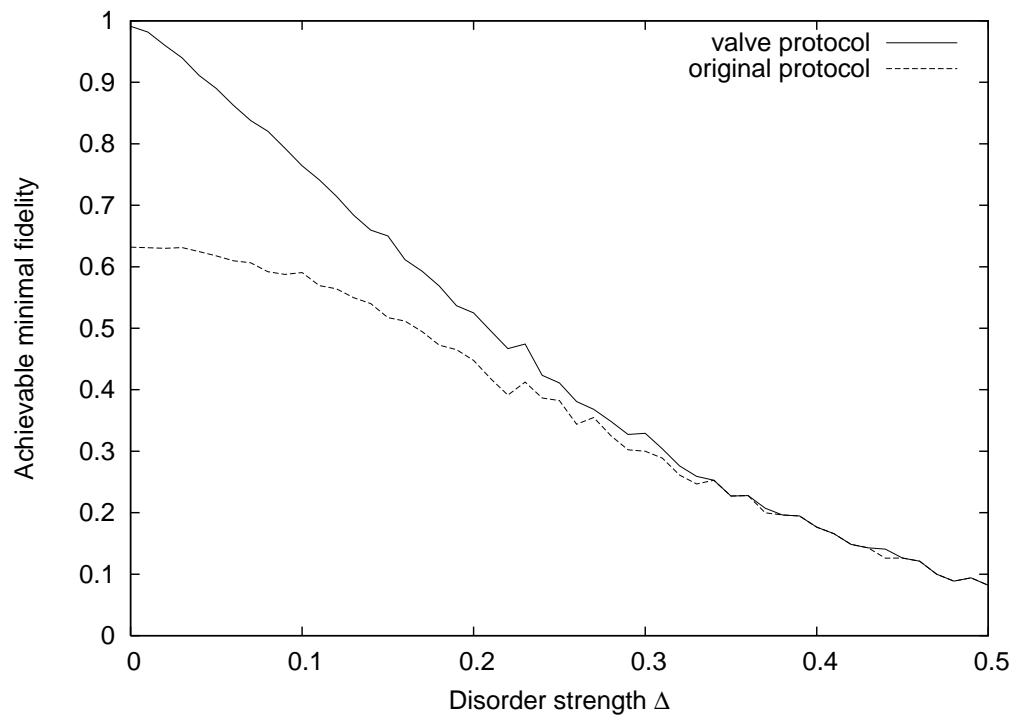

Fig. 6. Fidelity that can be achieved using maximally 20 steps of the valve protocol for a $X X$ chain of length $N=20$, i.e. $\max _{k<20} F_{0}^{k}$. Shown is the case where the ideal sequence is applied to unknown disorder as a function of the error strength $\Delta$ (averaged over 100 samples). As a comparison, we also give the fidelity $F_{0}$ of the original protocol [3] as a function of unknown disorder. Up to $\Delta \approx 0.3$ the fidelity can be improved using the valve.

\section{References}

1. D. Burgarth, V. Giovannetti, S. Bose (2006), quant-ph/0610018

2. H.P. Breuer, F. Petruccione, The Theory Of Open Quantum Systems (Oxford University Press, Oxford, 2002)

3. S. Bose, Phys. Rev. Lett. 91, 207901 (2003)

4. R. Cleve, J. Watrous, Annual Symposium on Foundations of Computer Science 41, 526 (2000) 
5. R. Jozsa (2006), quant-ph/0603163

6. N. Yoranand, A.J. Short (2006), quant-ph/0601178

7. H. Häffner, W. Hänsel, C.F. Roos, J. Benhelm, D. Chekalkar, M. Chwalla, T. Körber, U.D. Rapol, M. Riebe, P.O. Schmidt et al., Nature 438, 643 (2005)

8. A.J. Skinner, M.E. Davenport, B.E. Kane, Phys. Rev. Lett. 90, 087901 (2003)

9. D. Kielpinski, C. Monroe, D. Wineland, Nature 417, 709 (2002)

10. V. Subrahmanyam, A. Lakshminarayan, Phys. Lett. A 349, 164 (2006)

11. V. Subrahmanyam, Phys. Rev. A 69, 034304 (2004)

12. M.B. Plenio, J. Hartley, J. Eisert, New. J. Phys. 6, 36 (2004)

13. L. Amico, A. Osterloh, F. Plastina, R. Fazio, G.M. Palma, Phys. Rev. A 69, 022304 (2004)

14. M.J. Hartmann, M.E. Reuter, M.B. Plenio (2006), quant-ph/0608051

15. M.J. Hartmann, M.E. Reuter, M.B. Plenio, New. J. Phys. 8, 94 (2006)

16. F. Verstraete, M. Popp, J.I. Cirac, Phys. Rev. Lett. 92, 027901 (2004)

17. F. Verstraete, M.A. Martin-Delgado, J.I. Cirac, Phys. Rev. Lett. 92, 087201 (2004)

18. T. Boness, S. Bose, T.S. Monteiro, Phys. Rev. Lett. 96, 187201 (2006)

19. J. Fitzsimons, J. Twamley, Phys. Rev. A 72, 050301 (2005)

20. L.F. Santos, G. Rigolin, C.O. Escobar, Phys. Rev. A 69, 042304 (2004)

21. T. Boness, M. Stocklin, T. Monteiro (2006), quant-ph/0612074

22. J.P. Keating, N. Linden, J.C.F. Matthews, A. Winter (2006), quant-ph/0606205

23. T.J.G. Apollaro, F. Plastina, Phys. Rev. A 74, 062316 (2006)

24. C.K. Burrell, T.J. Osborne (2007), quant-ph/0703209

25. A. Romito, R. Fazio, C. Bruder, Phys. Rev. B 71, 100501(R) (2005)

26. A. Romito, G. de Chiara, D. Rossini and S. Montangero, Int. J. Quant. Inf., 4, 519 (2006)

27. D. Tsomokos, M. Hartmann, S. Huelga, M.B. Plenio, New. J. Phys. 9, 79 (2007)

28. A. Lyakhov, C. Bruder, Phys. Rev. B 74, 235303 (2006)

29. A. Lyakhov, C. Bruder, New. J. Phys. 7, 181 (2005)

30. D. Loss, D.P. DiVincenzo, Phys. Rev. A 57, 120 (1998)

31. A.D. Greentree, J.H. Cole, A.R. Hamilton, L.C.L. Hollenberg, Phys. Rev. B 70, 235317 (2004)

32. I. D'Amico (2005), cond-mat/0511470

33. T.P. Spiller, I. D'Amico, B.W. Lovett, New. J. Phys. 9, 20 (2006)

34. J. Zhang, X. Peng, D. Suter, Phys. Rev. A 73, 062325 (2006)

35. J. Fitzsimons, L. Xiao, S.C. Benjamin, J.A. Jones (2006), quant-ph/0606188

36. J. Zhang, G.L. Long, W. Zhang, Z. Deng, W. Liu, , Z. Lu, Phys. Rev. A 72, 012331 (2005)

37. J. Zhang, N. Rajendran, X .Peng and D. Suter, arXiv:0706.0352

38. J.J. Garcia-Ripoll, J.I. Cirac, New. J. Phys. 5, 76 (2003)

39. M. Paternostro, G.M. Palma, M.S. Kim, G. Falci, Phys. Rev. A 71, 042311 (2005)

40. M. Paternostro, Ph.D. thesis, The Queen's University of Belfast (2005)

41. D.G. Angelakis, M.F. Santos, S. Bose (2006), quant-ph/0606159

42. M.J. Hartmann, F.G.S.L. Brandao, M.B. Plenio, Nature Physics 2, 849 (2006)

43. S. Bose, D.G. Angelakis, D. Burgarth (2007), http://arxiv.org/abs/0704.0984

44. V. May, O. Kühn, Charge and Energy Transfer Dynamics in Molecular Systems (WileyInterscience, Hoboken, 2004)

45. N. Motoyama, H. Eisaki, S. Uchida, Phys. Rev. Lett. 76, 3212 (1996)

46. P. Gambardella, A. Dallmeyer, K. Maiti, M.C. Malagoli, W. Eberdardt, K. Kern, C. Carbone, Nature 416, 301 (2002)

47. A. Kay, Phys. Rev. Lett. 98, 010501 (2007)

48. H. Nakazato, T. Takazawa, K. Yuasa, Phys. Rev. Lett. 90, 060401 (2003)

49. D. Burgarth, V. Giovannetti (2007), arXiv:0704.3027

50. D. Burgarth, Ph.D. thesis, University College London (2006)

51. D. Kretschmann, R.F. Werner, Phys. Rev. A 72, 062323 (2005)

52. T.J. Osborne, N. Linden, Phys. Rev. A 69, 052315 (2004)

53. M. Christandl, N. Datta, T.C. Dorlas, A. Ekert, A. Kay, A.J. Landahl, Phys. Rev. A 71, 032312 (2005)

54. M. Christandl, N. Datta, A. Ekert, A.J. Landahl, Phys. Rev. Lett. 92, 187902 (2004)

55. G.M. Nikolopoulos, D. Petrosyan, P. Lambropoulos, Europhys. Lett. 65, 297 (2004)

56. R. Cook, B.W. Shore, Phys. Rev. A 20, 539 (1979)

57. A. Peres, Phys. Rev. A 32, 3266 (1985)

58. A. Kay, M. Ericsson, New. J. Phys. 7, 143 (2005)

59. M.H. Yung, S. Bose, Phys. Rev. A 71, 032310 (2005) 
60. M.H. Yung, Phys. Rev. A 74, 030303(R) (2006)

61. S. Yang, Z. Song, C.P. Sun, Phys. Rev. B 73, 195122 (2006)

62. Z. Song, C.P. Sun, Low Temp.Phys. 31, 907 (2005)

63. T. Shi, Y. Li, Z. Song, C.P. Sun, Phys. Rev. A 71, 032309 (2005)

64. D. Petrosyan, P. Lambropoulos, Opt.Commun. 264, 419 (2006)

65. G.M. Nikolopoulos, D. Petrosyan, P. Lambropoulos, J. Phys. C: Condens. Matter 16, 4991 (2004)

66. A. Kay, Phys. Rev. A 73, 032306 (2006)

67. A. Kay, Ph.D. thesis, University of Cambridge (2006), http://cam.qubit.org/users/Alastair/thesis.pdf

68. P. Karbach, J. Stolze, Phys. Rev. A 72, 030301(R) (2005)

69. L. Dan, Z. Jing-Fu, Chin.Phys. 15, 272 (2006)

70. C. Albanese, M. Christandl, N. Datta, A. Ekert, Phys. Rev. Lett. 93, 230502 (2004)

71. V. Kostak, G. M. Nikolopoulos and I. Jex, Phys. Rev. A 75, 042319 (2007).

72. M.B. Plenio, F.L. Semião, New. J. Phys. 7, 73 (2005)

73. A. Wójcik, T. Łuczak, P. Kurzyński, A. Grudka, T. Gdala, M. Bednarska (2006), quant-ph/0608107

74. A. Wójcik, T. Łuczak, P. Kurzyński, A. Grudka, T. Gdala, M. Bednarska, Phys. Rev. A 72, 034303 (2005)

75. Y. Li, T. Shi, B. Chen, Z. Song, C.P. Sun, Phys. Rev. A 71, 022301 (2005)

76. M. Avellino, A.J. Fisher, S. Bose, Phys. Rev. A 74, 012321 (2006)

77. M. Avellino, A.J. Fisher, S. Bose, Phys. Rev. A 74, 039901 (2006)

78. S. Paganelli, F. de Pasquale, G.L. Giorgi, Phys. Rev. A 74, 012316 (2006)

79. L.C. Venuti, C.D.E. Boschi, M. Roncaglia (2007), quant-ph/0703202

80. L.C. Venuti, C.D.E. Boschi, M. Roncaglia, Phys. Rev. Lett. 96, 247206 (2006)

81. G. De Chiara, D. Rossini, S. Montangero and R. Fazio, Phys. Rev. A 72, 012323 (2005)

82. L. Dan and Z. Jing-Fu, Chin. Phys. 15, 272 (2006)

83. D. Burgarth, S. Bose, Phys. Rev. A 71, 052315 (2005)

84. D. Burgarth, S. Bose, New. J. Phys. 7, 135 (2005)

85. D. Burgarth, V. Giovannetti, S. Bose, J. Phys. A: Math. Gen. 38, 6793 (2005)

86. V. Giovannetti, D. Burgarth, Phys. Rev. Lett. 96, 030501 (2006)

87. H.L. Haselgrove, Phys. Rev. A 72, 062326 (2005)

88. J. Fitzsimons, J. Twamley, Phys. Rev. Lett. 97, 090502 (2006)

89. R. Raussendorf, Phys. Rev. A 72, 052301 (2005)

90. S. Yang, Z. Song, C.P. Sun, Phys. Rev. A 73, 022317 (2006)

91. K. Maruyama, T. Iitaka, F. Nori (2006), quant-ph/0610103

92. S. Bose, B. Jin, V.E. Korepin, Phys. Rev. A 72, 022345 (2005)

93. S. Yang, Z. Song, C. Sun, Front. Phys. China 1, 1 (2007)

94. K. Eckert, O. Romero-Isart, A. Sanpera (2007), quant-ph/0702082 